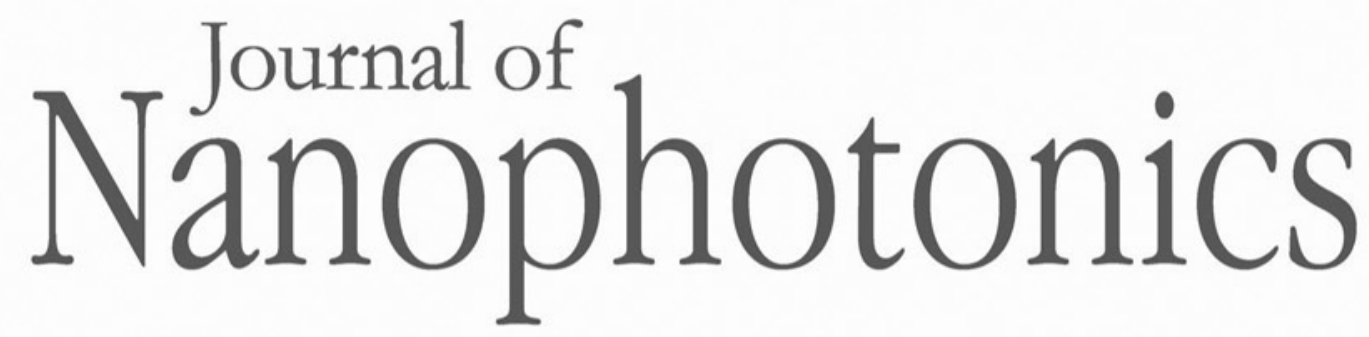

\title{
Picosecond optical vortex-induced chiral surface relief in an azo- polymer film
}

\author{
Keigo Masuda \\ Ryo Shinozaki \\ Ami Shiraishi \\ Mitsuki Ichijo \\ Keisaku Yamane \\ Katsuhiko Miyamoto \\ Takashige Omatsu
}




\title{
Picosecond optical vortex-induced chiral surface relief in an azo-polymer film
}

\author{
Keigo Masuda, ${ }^{a}$ Ryo Shinozaki, ${ }^{a}$ Ami Shiraishi, ${ }^{a}$ Mitsuki Ichijo, ${ }^{\text {a }}$ \\ Keisaku Yamane, ${ }^{b}$ Katsuhiko Miyamoto, ${ }^{\text {a,c }}$ and Takashige Omatsu ${ }^{\text {a,c,* }}$ \\ ${ }^{a}$ Chiba University, Graduate School of Engineering, Chiba, Japan \\ ${ }^{b}$ Hokkaido University, Department of Applied Physics, Sapporo, Japan \\ ${ }^{\mathrm{C}}$ Chiba University, Molecular Chirality Research Center, Chiba, Japan
}

\begin{abstract}
We report on the first demonstration of picosecond optical vortex-induced chiral surface relief in an azo-polymer film due to two-photon absorption isomerization. The chiral surface relief exhibits an extremely narrow defocusing tolerance without undesired outer rings due to the Airy pattern of highly focused light. Such chiral surface relief reflects a $z$-polarized electric field with an azimuthal helical phase caused by spin-orbital angular momentum coupling. () The Authors. Published by SPIE under a Creative Commons Attribution 4.0 Unported License. Distribution or reproduction of this work in whole or in part requires full attribution of the original publication, including its DOI. [DOI: 10.1117/1.JNP.14.016012]
\end{abstract}

Keywords: singular optics; optical vortices; orbital angular momentum; spin angular momentum; laser materials processing; chiral structured materials; azopolymers.

Paper 19144SS received Nov. 22, 2019; accepted for publication Mar. 3, 2020; published online Mar. 18, 2020.

\section{Introduction}

Azo polymers enable photoinduced reversible surface relief to be realized; ${ }^{1}$ therefore, they will play an important role in the realization of rewritable optical data storage ${ }^{2-4}$ and light-driven micro/nanorobots. ${ }^{5-7}$ The formation of surface relief in azo polymers generally occurs through several steps: thermodynamically stable trans-form azo polymers are mostly stable at room temperature. ${ }^{8} 9$ Irradiation of trans-form azo polymers with nonuniform light leads to nonuniform trans-cis photoisomerization of azo polymers via light absorption and subsequent reversion of the azo polymers to the trans-form by spontaneous thermal reaction and the cis-trans reverse photoisomerization. ${ }^{10-13}$ This trans-cis/cis-trans photoisomerization cycle (typical cycle time of 1 to $2 \mathrm{ps)} \mathrm{forces} \mathrm{a} \mathrm{spring-like} \mathrm{motion} \mathrm{of} \mathrm{the} \mathrm{azo} \mathrm{polymers,} \mathrm{which} \mathrm{results} \mathrm{in} \mathrm{spatially} \mathrm{anisotropic}$ photofluidity. ${ }^{14-17}$ The mass transport of azo polymers thus occurs from a bright region toward a dark region along the polarization direction of the irradiated light by the gradient force based on interaction between photo-oriented azo polymers and the gradient of an optical electric field, i.e., light-induced mass transport, which results in the establishment of surface relief. The structures of the surface relief are thus mostly determined by the spatial intensity profile and polarization of the irradiated light. ${ }^{18-21}$ Optical vortices carry an annular intensity profile and orbital angular momentum (OAM), characterized by a quantum number $\ell$, which associated with its helical wavefront. ${ }^{22-26}$ To date, several researchers have reported that irradiation with a continuous-wave visible optical vortex can force the helical mass transport of irradiated azo polymers to form wavefront-sensitive surface relief, i.e., chiral surface relief, facilitated by the spin angular momentum (SAM) of circularly polarized light with a helical electric field. ${ }^{27-31}$

An ultrafast laser-induced two-photon-absorption (TPA) process offers the trans-cis photoisomerization of azo polymers with high spatial resolution, which could yield further advanced technologies, such as high-density rewritable data storage. ${ }^{32-34}$ However, most studies on surface relief formation in azo polymers are still based on single-photon-absorption (SPA). ${ }^{1,8-21,27-11}$ Surface relief formation via TPA has not yet been well established. Furthermore, there have been no reports on optical vortex-induced surface relief formation in azo polymers via TPA

*Address all correspondence to Takashige Omatsu, E-mail: omatsu@faculty.chiba-u.jp 
isomerization. Optical vortex-induced TPA will provide not only the helical mass transport of azo polymers for high-density rewritable data storage with a freedom of $\mathrm{OAM}^{35-37}$ but also entirely new nonlinear and photochemical phenomena, such as an OAM conservation law in TPA, and spin and OAM coupling in TPA. ${ }^{38-40}$

Here we report on TPA-induced chiral surface relief formation by irradiation with a nearinfrared picosecond optical vortex.

\section{Experiments}

\subsection{Two-Photon-Absorption Induced Surface Relief}

A 1- $\mu \mathrm{m}$ thick azo-polymer (poly-orange tom-1) thin film spin-coated on a slide glass was used in the present experiments, which exhibited strong absorption at $532 \mathrm{~nm}$ (no absorption at $1.06 \mu \mathrm{m}$ ), as shown in Fig. 1(a). Therefore, two-photon isomerization should deform the azo-polymer film. A $1.06-\mu \mathrm{m}$ picosecond laser with a pulse width of $8 \mathrm{ps}$, a pulse repetition frequency of $35 \mathrm{MHz}$, and an average power of $40 \mathrm{~mW}$ was used as a laser source, and its output was converted into a right-handed circularly polarized optical vortex with a total angular momentum of $J=2$ by employing a spiral phase plate (OAM: $\ell=1$ ) and a quarter wave plate (SAM: $s=1$ ). The generated vortex beam was focused to be an annular spot with a diameter of $3.6 \mu \mathrm{m}$ onto the azo-polymer film surface from the front side, i.e., the atmosphere side, using a high numerical aperture (NA) objective lens $(\mathrm{NA}=0.9)$ [Fig. 1(b)]. The corresponding intensity of the focused vortex beam and the exposure time were then $\sim 1.4 \mathrm{GW} / \mathrm{cm}^{2}$ and $300 \mathrm{~s}$ (dose energy of $12 \mathrm{~J}$ ), respectively.

The optical vortex caused the upward mass transport of azo polymers along the beam propagation axis ( $z$ axis). The azo polymers were subsequently directed inward toward the dark core of the optical vortex and revolved along the clockwise direction to establish single-armed chiral surface relief, which reflects the helical wavefront of the optical vortex field within $120 \mathrm{~s}(4.8 \mathrm{~J})$ [Fig. 2(a)]. The chiral surface relief formed had a diameter of $3.5 \mu \mathrm{m}$ and a height of $\sim 300 \mathrm{~nm}$. The formation of chiral surface relief on the azo polymers was difficult for an optical vortex with a dose energy of $<\sim 3 \mathrm{~J}$, whereas the SPA-induced helical mass transport of azo polymers can

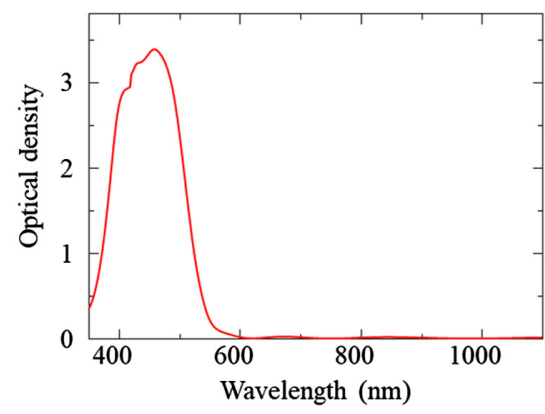

(a)

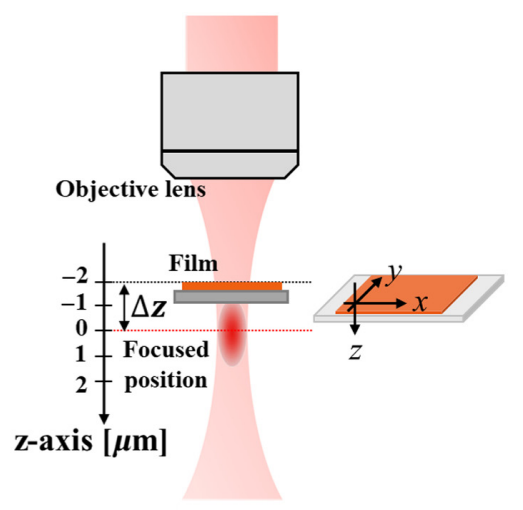

(b)

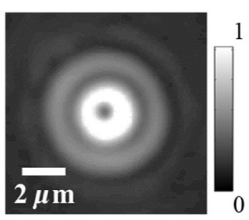

(c)

Fig. 1 (a) Absorption spectrum of the azo-polymer film used in the present experiment. (b) Experimental-setup. (c) Experimental spatial profile of the strongly focused optical vortex. The focused optical vortex exhibits multiple rings due to Airy pattern. 

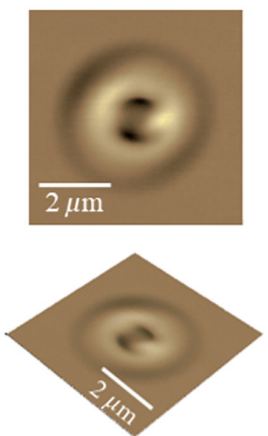

$60 \mathrm{~s}(2.4 \mathrm{~J})$
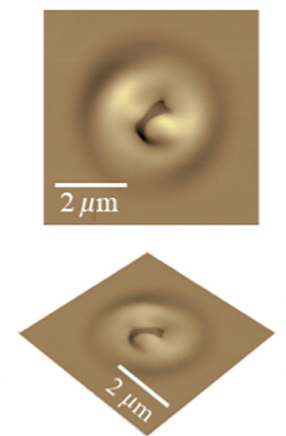

$120 \mathrm{~s}(4.8 \mathrm{~J})$
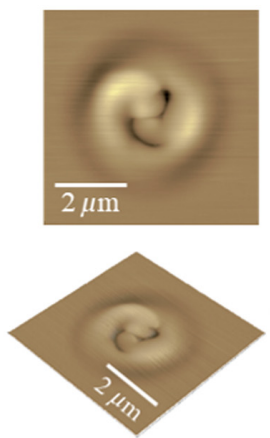

$180 \mathrm{~s}(7.2 \mathrm{~J})$
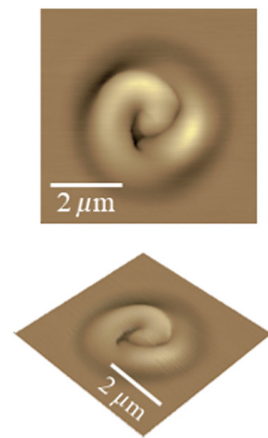

$300 \mathrm{~s}(12 \mathrm{~J})$

(a)
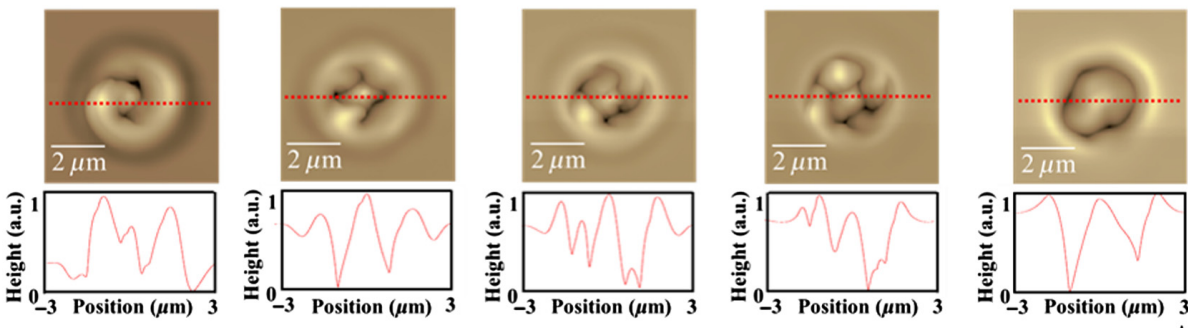

$-2 \mu \mathrm{m}$

$-1 \mu \mathrm{m}$

$0 \mu \mathrm{m}$

$1 \mu \mathrm{m}$

$2 \mu \mathrm{m}$

(b)

Fig. 2 (a) Temporal evolution of surface relief formation in an azo-polymer film by irradiation with 8 ps optical vortex pulses with $J=2$. (b) Atomic force microscopy (AFM) images of surface relief produced with various defocusing depths. Chiral relief can be formed only at a defocusing depth $\Delta z$ of $-2 \mu \mathrm{m}$. The lower panels show line profiles of surface relief along the red lines in the AFM images.

occur with only a dose energy of $\sim 2 \mathrm{~mJ}$, as previously reported. ${ }^{28-30}$ The TPA-induced mass transport of azo polymers ${ }^{41}$ is enabled by a longitudinal gradient of light intensity of the focused spot only within a small displacement of focused position from the film along $z$ axis, i.e., defocusing depth $\Delta z$, as defined in Fig. 1(b). In fact, the formation of chiral surface relief occurred only at the defocusing depth $\Delta z$ of $-2 \mu \mathrm{m}$, which indicates that this is TPA-induced photoisomerization [Fig. 2(b)]. The strongly focused optical vortex, in general, exhibits multiple rings owing to the Airy pattern [Fig. 1(c)]. However, the created surface relief is shaped to be a single-armed ring without outer rings that also manifests strongly TPA photoisomerization with significant optical intensity threshold.

The optical vortex pulse was compressed to $\sim 2$ ps without any change of the peak intensity (average power $\sim 10 \mathrm{~mW}$ ). The optical vortex then typically induced a ring-shaped (not well twisted) surface relief, which mostly reflects the spatial intensity profile of the focused optical vortex field [Fig. 3(a)]. A ring-shaped surface relief (although slightly asymmetric) was produced even with an exposure time of $1200 \mathrm{~s}$.

It is further noteworthy that the optical vortex with an even higher peak intensity induced photon-bleaching of the azo polymer and ablation of the film itself, which resulted in the formation of an abnormal ring-shaped surface relief with two petals [Fig. 3(b)].

These results further indicate that the helical mass transport, i.e., chiral surface relief formation, requires an optical vortex with a pulse duration of at least several photoisomerization cycles. The results of experiments performed by irradiation with optical vortices with various pulse durations are summarized in Fig. 4.

An optical vortex with $J=0$ was also generated by inversion of the quarter wave plate, which produced only a nonhelical single damp-shaped surface relief, regardless of the pulse duration and exposure time (Fig. 5). 


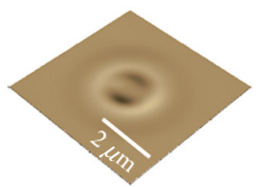

$240 \mathrm{~s}(2.4 \mathrm{~J})$

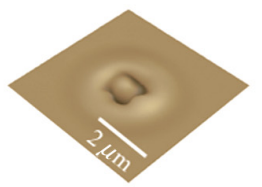

$480 \mathrm{~s}(4.8 \mathrm{~J})$

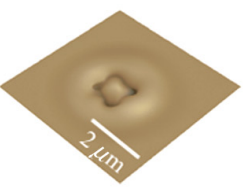

$720 \mathrm{~s}(7.2 \mathrm{~J})$

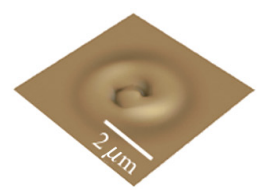

$1200 \mathrm{~s}(12 \mathrm{~J})$

(a)
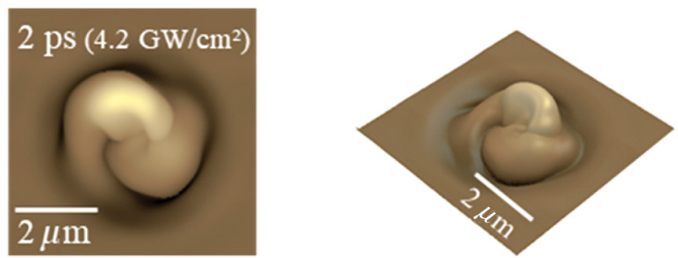

(b)

Fig. 3 (a) Surface relief formation in azo-polymer film by irradiation with a 2-ps optical vortex with $J=2$. The surface relief formed was mostly ring-shaped (not well twisted). (b) Surface relief with two petals was formed by a 2-ps optical vortex. The intensity of the focused optical vortex was measured to be $4.2 \mathrm{GW} / \mathrm{cm}^{2}$.
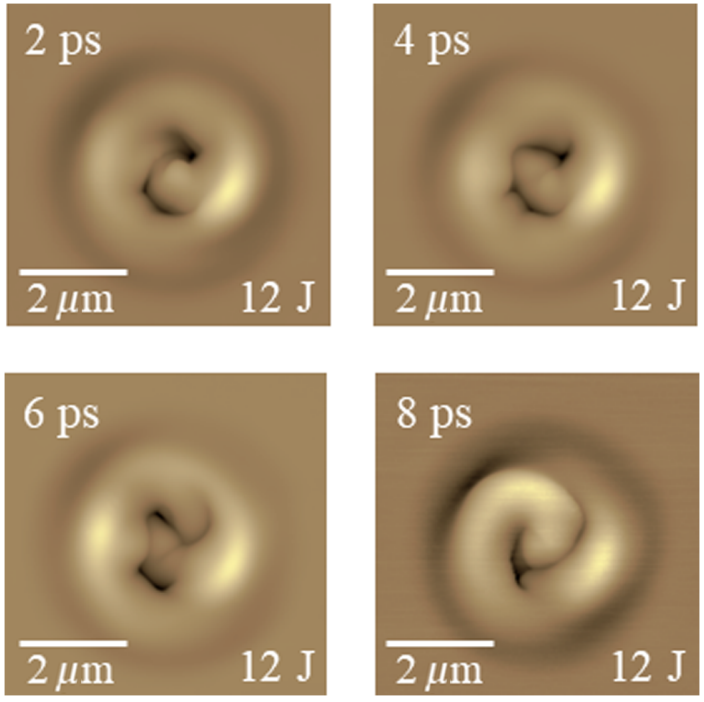

Fig. 4 TPA-induced surface relief formed by optical vortices with various pulse durations (2, 4, 6, and 8 ps).

\subsection{Single-Photon-Absorption Induced Surface Relief}

We also investigated SPA-induced surface relief formation by employing a 532-nm optical vortex with a pulse width of $8 \mathrm{ps}$, a pulse repetition frequency of $35 \mathrm{MHz}$, and an average power of $3 \mu \mathrm{W}$. The optical vortex was focused to be an annular spot with a diameter of $3.6 \mu \mathrm{m}$ at the focal plane; the corresponding intensity was estimated to be $105 \mathrm{~kW} / \mathrm{cm}^{2}$ and the exposure time was $300 \mathrm{~s}$ (dose energy: $0.9 \mathrm{~mJ}$ ). The irradiated azo polymers also exhibited surface relief that was directed in a clockwise direction [Fig. 6(a)]. However, the resultant chiral surface relief had outer rings due to the Airy pattern of the strongly focused optical vortex. The height of the surface relief was typically measured to be $100 \mathrm{~nm}$. Chiral surface relief was further formed at any defocusing depth $\Delta z$ within the range of -2 to $+2 \mu \mathrm{m}$ [Fig. 6(b)]. These results indicate that TPA-induced photoisomerization enables the formation of chiral surface relief in azo polymers with high longitudinal and transverse spatial resolution [Fig. 6(c)]. 

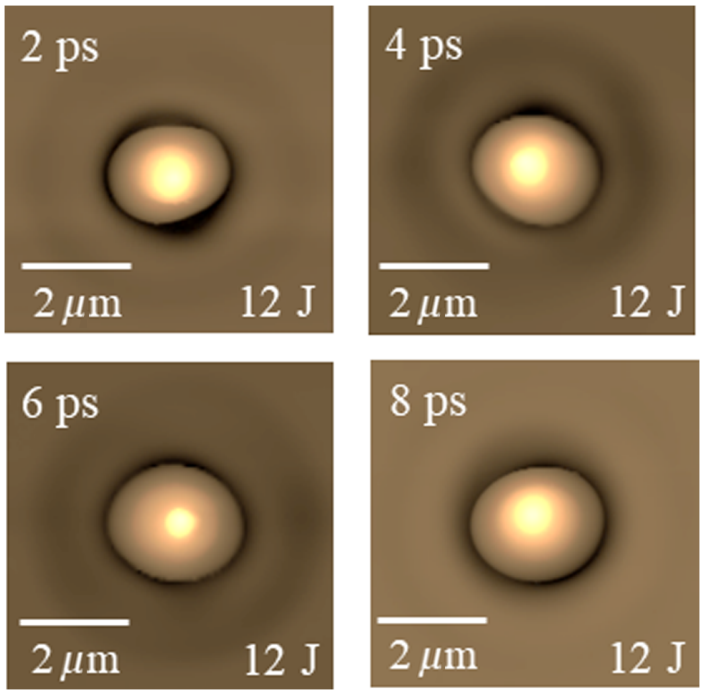

Fig. 5 TPA-induced surface relief formed by optical vortices $(J=0)$ with various pulse durations.

It should be noted that a 2-ps optical vortex did not induce efficient helical mass transport of the azo polymer [Fig. 6(d)]. Therefore, the helical mass transport of the azo polymer, even via SPA, requires an optical vortex with a pulse duration of at least several photoisomerization cycles.

\section{Discussion}

The experiments on TPA and SPA suggest that TPA contributes primarily to photoisomerization rather than mass transport of azo polymers. Here we discuss the surface relief formation based on classical optical radiation force based on time-averaged Poynting vector.

The electromagnetic fields of the circularly polarized optical vortex strongly focused by a lens with a high NA $\left(=\sin \theta_{\max }\right)>0.8$ can be analyzed using the theoretical formula as the Debye vector integral: ${ }^{42}$

$$
\begin{aligned}
\mathbf{E}(r, \varphi, z)= & {\left[\begin{array}{c}
E_{x} \\
E_{y} \\
E_{z}
\end{array}\right]=-\frac{i k f}{2 \pi} \int_{0}^{\theta_{\max }} \int_{0}^{2 \pi}\left(\frac{\sqrt{2} f \sin \theta}{w}\right)^{|\ell|} \exp \left(-\frac{f^{2} \sin ^{2} \theta}{w^{2}}\right) \sin \theta \sqrt{\cos \theta} \exp (i \ell \phi) } \\
& \times\left[\begin{array}{c}
\left(\cos ^{2} \phi \cos \theta+\sin ^{2} \phi\right) \pm i \cos \phi \sin \phi(\cos \theta-1) \\
\cos \phi \sin \phi(\cos \theta-1) \pm i\left(\cos ^{2} \phi+\sin ^{2} \phi \cos \theta\right) \\
\sin \theta \exp ( \pm i \phi)
\end{array}\right] \mathrm{e}^{i k[z \cos \theta+r \sin \theta \cos (\phi-\varphi)]} \mathrm{d} \phi \mathrm{d} \theta
\end{aligned}
$$

where $E_{x}, E_{y}$, and $E_{z}$ are the $x$-, $y$-, and $z$-polarized electric fields in the focused plane; $f$ is the focal distance; $w$ is the beam waist; $k$ is the wave vector; and $r, \varphi, \theta$, and $\phi$ are shown in Fig. 7(a). The circularly polarized optical vortex with $J=2$ produces an annular amplitude profile and a $4 \pi$ azimuthal phase shift due to constructive spin-orbital coupling effects in an isotropic and homogeneous material, thereby yielding an annular focused spot, which is defined as the sum of the transverse and $z$-polarized electric fields ${ }^{43-46}$ [Figs. 7(b)-7(d)]. The resulting scattering force on the azo-polymer film, given as ${ }^{29}$

$$
\mathbf{F}_{\text {scattering }}=\left\langle-i \omega \varepsilon_{0} \chi \mathbf{E} \times \mathbf{B}\right\rangle=\frac{\omega \varepsilon_{0}}{2}\left[\chi_{r} \operatorname{Im}\left(\mathbf{E} \times \mathbf{B}^{*}\right)+\chi_{i} \operatorname{Re}\left(\mathbf{E} \times \mathbf{B}^{*}\right)\right]
$$




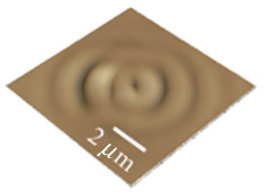

$60 \mathrm{~s}(0.18 \mathrm{~mJ})$

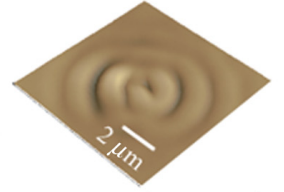

$120 \mathrm{~s}(0.36 \mathrm{~mJ})$

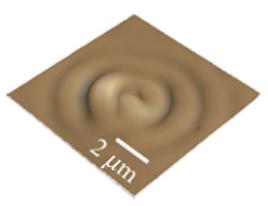

$180 \mathrm{~s}(0.54 \mathrm{~mJ})$

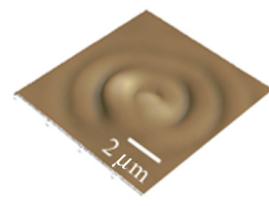

$300 \mathrm{~s}(0.9 \mathrm{~mJ})$

(a)
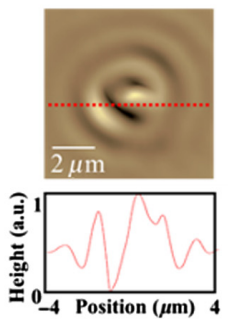

$-2 \mu \mathrm{m}$
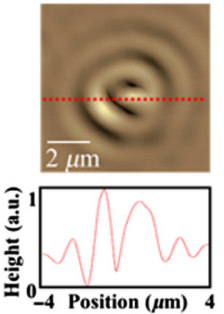

$-1 \mu \mathrm{m}$
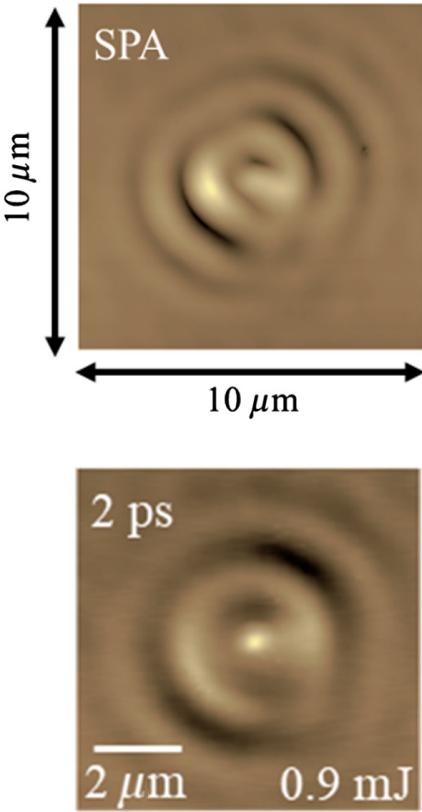
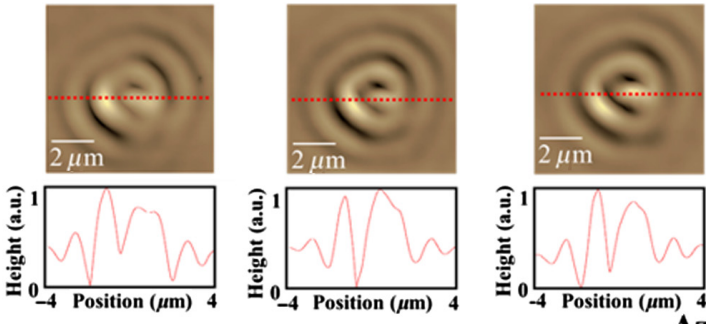

$1 \mu \mathrm{m}$

$0 \mu \mathrm{m}$

(b)

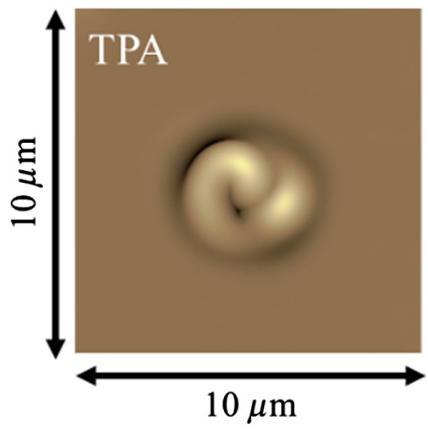

(c)

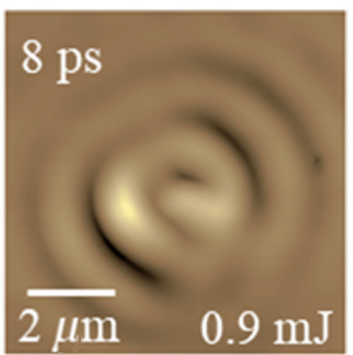

(d)

Fig. 6 (a) Temporal evolution of surface relief formation in an azo-polymer film by irradiation with $532 \mathrm{~nm} 8$ ps optical vortex pulses with $J=2$. (b) AFM images of surface relief formed with various defocusing depths. Chiral relief is formed at any defocusing depth $\Delta z$ within the range of -2 to $+2 \mu \mathrm{m}$. (c) SPA or TPA induced chiral surface relief. TPA produces chiral relief without any undesired outer rings due to the Airy pattern. (d) Surface relief formed by $532 \mathrm{~nm}$ optical vortices with pulse durations of 2 and 8 ps.

twists and directs the azo polymer toward the dark core with the assistance of the gradient force $\left(F_{\text {gradient }}=-\mathrm{d} I / \mathrm{d} r\right)$, so as to form a ring-shaped helical surface relief [Fig. 7(h)].

In contrast, an optical vortex with $J=0$ creates a theoretically focused spot with a central bright spot due to destructive spin-orbital coupling effects [Figs. 7(e)-7(g)], thereby yielding a damp-shaped relief via TPA induced photoisomerization [Fig. 7(i)]. These analyses support the observed experimental surface relief. Though it is beyond the scope of this paper, further modeling of optical radiation force, including TPA effects, should be investigated as a future work. 


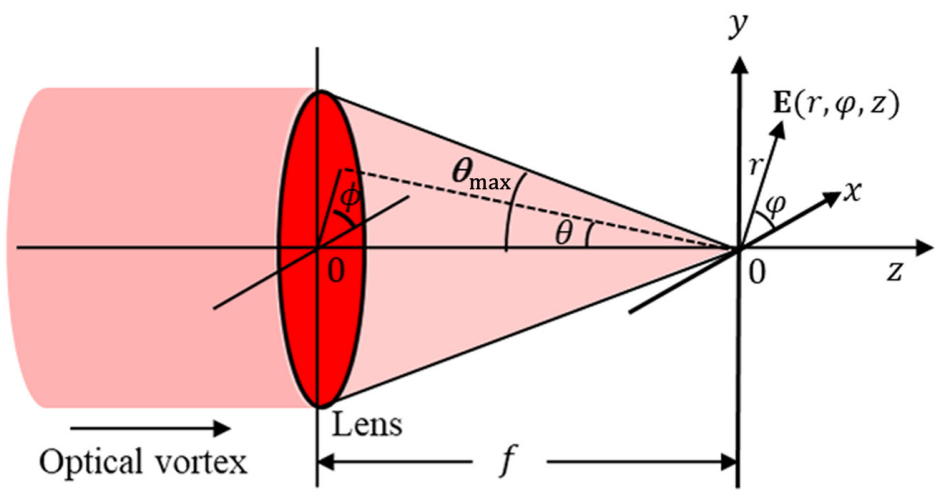

(a)
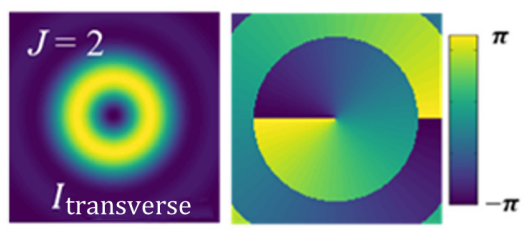

(b)
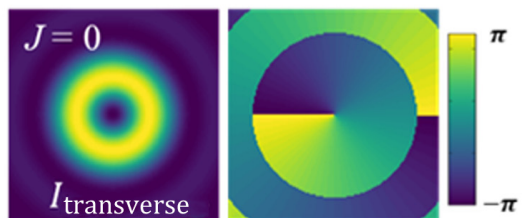

(e)

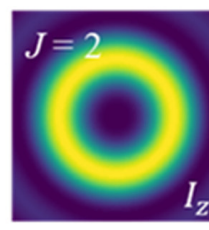

(c)

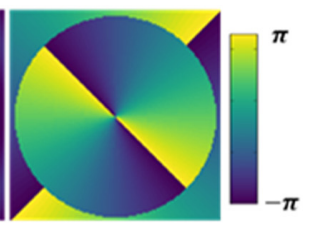

c)

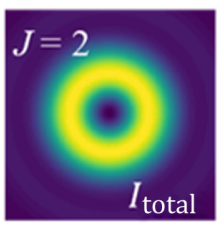

(d)

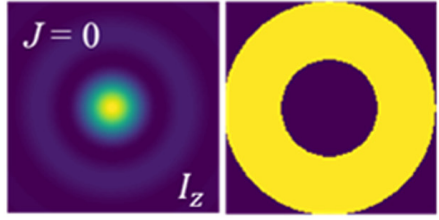

(f)

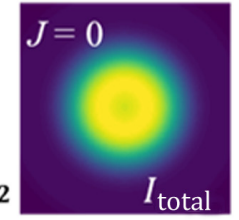

(g)

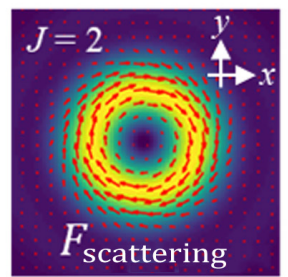

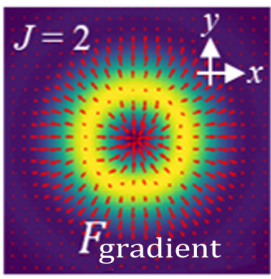

(h)

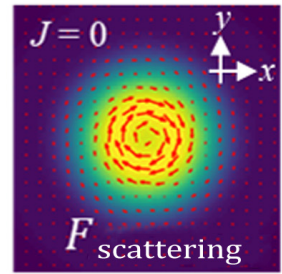

(i)

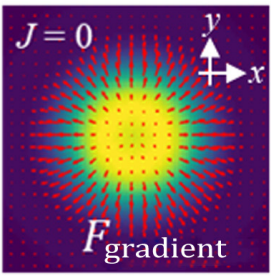

Fig. 7 (a) The coordinate system for strongly focused optical vortex. Theoretical spatial intensity profiles and phases of (b), (e) transverse (in the focused plane) and (c), (f) z-polarized electric fields of strongly focused circularly polarized optical vortices with $J=2$ and $J=0$. (d), (g) Spatial intensity profiles of strongly focused circularly polarized optical vortices with $J=2$ and $J=0$. Optical scattering and gradient forces created by strongly focused optical vortices with (h) $J=2$ and (i) $J=0$.

\section{Conclusion}

We have successfully demonstrated the formation of TPA-induced chiral surface relief in an azo-polymer film for the first time by employing a picosecond optical vortex source. The TPAinduced photoisomerization allows the formation of chiral surface relief with high longitudinal and transverse spatial resolution, i.e., without undesired outer rings due to Airy patterns and only within an extremely narrow defocusing tolerance. The formation of such surface relief also requires an optical vortex with a pulse duration of at least several trans-cis/cistrans photoisomerization cycles. An optical vortex with a pulse duration of $<6$ ps impacted the formation of chiral surface relief with the use of any focusing region and exposure time. The SAM-OAM coupling effects create a strong $z$-polarized electric field with an azimuthal phase, which contributes significantly to the helical mass transport of the azo polymer. 
Such TPA-induced helical mass transport is expected to lead to the realization of high-density optical data storage with the freedom of OAM and offer entirely new physical insights for the interplay between structured light fields and materials.

\section{Acknowledgments}

The authors acknowledge the financial support in the form of Kakenhi Grants-in-Aid (Nos. JP16H06507, JP17K19070, and JP18H03884) from the Japan Society for the Promotion of Science (JSPS) and a CREST grant (No. JPMJCR1903) from the Japan Science and Technology Agency (JST).

\section{References}

1. N. K. Viswanathan et al., "Surface relief structures on azo polymer films," J. Mater. Chem. 9(9), 1941-1955 (1999).

2. G. Xu et al., "Application of all-optical poling in reversible optical storage in azopolymer films," Opt. Commun. 159(1-3), 88-92 (1999).

3. W. Zhang et al., "High-efficiency holographic volume index gratings in DR1-dye-doped poly(methyl methacrylate)," Opt. Lett. 27(13), 1105-1107 (2002).

4. D. Gindre et al., "Rewritable optical data storage in azobenzene copolymers," Opt. Express 14(21), 9896-9901 (2006).

5. Y. Yu, M. Nakano, and T. Ikeda, "Directed bending of a polymer film by light," Nature 425, 145-145 (2003).

6. T. Muraoka et al., "Light-driven open-close motion of chiral molecular scissors," J. Am. Chem. Soc. 125(19), 5612-5613 (2003).

7. J. J. Wie, M. R. Shankar, and T. J. White, "Photomotility of polymers," Nat. Commun. 7, 13260 (2016).

8. A. Natansohn and P. Rochon, "Reversible optical storage in azo polymers," in Progress in Pacific Polymer Science, K. P. Ghiggino, Ed., Vol. 3, pp. 295-305, Springer, Berlin, Heidelberg (1994).

9. M. S. Ho et al., "Azo polymers for reversible optical storage. 8. The effect of polarity of the azobenzene groups," Can. J. Chem. 73(11), 1773-1778 (1995).

10. H. Rau, "Photoisomerization of benzenes," in Photoreactive Organic Thin Films, Z. Sekkat and W. Knoll, Eds., 1st ed., pp. 3-28, Academic Press, Cambridge, Massachusetts (2002).

11. Z. Sekkat, "Photo-orientation by photoisomerization," in Photoreactive Organic Thin Films, Z. Sekkat and W. Knoll, Eds., 1st ed., pp. 64-102, Academic Press (2002).

12. X. Wang, Ed., "Trans-cis isomerization," in Azo Polymers, 1st ed., pp. 19-56, Springer, Berlin, Heidelberg, Germany (2016).

13. T. Fujino, S. Y. Arzhantsev, and T. Tahara, "Femtosecond time-resolved fluorescence study of photoisomerization of trans-azobenzene," J. Phys. Chem. A 105(35), 8123-8129 (2001).

14. Z. Sekkat, "Optical tweezing by photomigration," Appl. Opt. 55(2), 259-268 (2016).

15. H. Ishitobi et al., "The anisotropic nanomovement of azo-polymers," Opt. Express 15(2), 652-659 (2007).

16. P. Karageorgiev et al., "From anisotropic photo-fluidity towards nanomanipulation in the optical near-field," Nat. Mater. 4(9), 699-703 (2005).

17. P. Lefin, C. Fiorini, and J. M. Nunzi, "Anisotropy of the photoinduced translation diffusion of azo-dyes," Opt. Mater. 9(1-4), 323-328 (1998).

18. J. Kumar et al., "Gradient force: the mechanism for surface relief grating formation in azobenzene functionalized polymers," Appl. Phys. Lett. 72(17), 2096-2098 (1998).

19. C. J. Barrett, A. L. Natansohn, and P. L. Rochon, "Mechanism of optically inscribed highefficiency diffraction gratings in azo polymer films," J. Phys. Chem. 100(21), 8836-8842 (1996).

20. P. Rochon, E. Batalla, and A. Natansohn, "Optically induced surface gratings on azoaromatic polymer films," Appl. Phys. Lett. 66(2), 136-138 (1995).

21. A. Kravchenko et al., "Optical interference lithography using azobenzene-functionalized polymers for micro-and nanopatterning of silicon," Adv. Mater. 23(36), 4174-4177 (2011). 
22. L. Allen et al., "Orbital angular momentum of light and the transformation of LaguerreGaussian laser modes," Phys. Rev. A 45(11), 8185-8189 (1992).

23. M. Padgett, J. Courtial, and L. Allen, "Light's orbital angular momentum," Phys. Today 57(5), 35-40 (2004).

24. X. Wang et al., "Recent advances on optical vortex generation," Nanophotonics 7(9), 1533 1556 (2018).

25. Y. Shen et al., "Optical vortices 30 years on: OAM manipulation from topological charge to multiple singularities," Light Sci. Appl. 8, 90 (2019).

26. T. Omatsu et al., "A new twist for materials science: the formation of chiral structures using the angular momentum of light," Adv. Opt. Mater. 7(14), 1801672 (2019).

27. A. Ambrosio et al., "Light-induced spiral mass transport in azo-polymer films under vortexbeam illumination," Nat. Commun. 3, 989 (2012).

28. M. Watabe et al., "Light induced conch-shaped relief in an azo-polymer film," Sci. Rep. 4, 4281 (2015).

29. D. Barada et al., "Constructive spin-orbital angular momentum coupling can twist materials to create spiral structures in optical vortex illumination," Appl. Phys. Lett. 108(5), 051108 (2016).

30. K. Masuda et al., "Azo-polymer film twisted to form a helical surface relief by illumination with a circularly polarized Gaussian beam," Opt. Express 25(11), 12499-12507 (2017).

31. K. Masuda et al., "Nanoscale chiral surface relief of azo- polymers with nearfield OAM light," Opt. Express 26(17), 22197-22207 (2018).

32. S. Maruo, O. Nakamura, and S. Kawata, "Three-dimensional microfabrication with two-photon-absorbed photopolymerization," Opt. Lett. 22(2), 132-134 (1997).

33. H. B. Sun and S. Kawata, "Two-photon photopolymerization and 3D lithographic microfabrication," in NMR · 3D Analysis · Photopolymerization, Advances in Polymer Science, K. S. Lee, Ed., Vol. 170, pp. 169-273, Springer, Berlin, Heidelberg, Germany (2004).

34. Z. Sekkat, "Vectorial motion of matter induced by light fueled molecular machines," OSA Continuиm 1(2), 668-681 (2018).

35. M. Gu et al., "Effect of saturable response to two-photon absorption on the readout signal level of three-dimensional bit optical data storage in a photochromic polymer," Appl. Phys. Lett. 79(2), 148-150 (2001).

36. R. J. Voogd, M. Singh, and J. J. M. Braat, "The use of orbital angular momentum of light beams for optical data storage," Proc. SPIE 5380, 387-392 (2004).

37. M. Gu, X. Li, and Y. Cao, "Optical storage arrays: a perspective for future big data storage," Light Sci. Appl. 3, e177 (2014).

38. K. Y. Bliokh et al., "Spin-orbit interactions of light," Nat. Photonics 9(12), 796-808 (2015).

39. S. Franke-Arnold, "Optical angular momentum and atoms," Philos. Trans. R. Soc. A 375, 20150435 (2017).

40. S. Ishii, N. Yokoshi, and H. Ishihara, "Optical selection rule of monolayer transition metal dichalcogenide by an optical vortex," J. Phys. Conf. Ser. 1220, 012056 (2019).

41. H. Ishitobi et al., "Two-photon induced polymer nanomovement," Opt. Express 16(18), 14106-14114 (2008).

42. Y. Zhao et al., "Spin-to-orbital angular momentum conversion in a strongly focused optical beam," Phys. Rev. Lett. 99(7), 15-18 (2007).

43. B. Richards and E. Wolf, "Electromagnetic diffraction in optical systems, II. Structure of the image field in an aplanatic system," Proc. R. Soc. London Ser. A 253(1274), 358-379 (1959).

44. L. Cicchitelli, H. Hora, and R. Postle, "Longitudinal field components for laser beams in vacuum," Phys. Rev. A 41(7), 3727-3732 (1990).

45. L. Novotny et al., "Longitudinal field modes probed by single molecules," Phys. Rev. Lett. 86(23), 5251-5254 (2001).

46. E. Yew and C. Sheppard, "Second harmonic generation polarization microscopy with tightly focused linearly and radially polarized beams," Opt. Coтmun. 275(2), 453-457 (2007).

Keigo Masuda received his BE and ME degrees from Chiba University in 2016 and 2018, respectively. $\mathrm{He}$ is currently studying materials processing and manipulation. $\mathrm{He}$ is also a member of OSA, JSAP, and SPIE. 
Ryo Shinozaki received his BE degree from Chiba University in 2018. He is currently working on surface relief formation of azo polymers.

Ami Shiraishi is an undergraduate student at Chiba University. She currently joins a project of optical materials processing and manipulation.

Mitsuki Ichijo received her BE degree from Chiba University in 2018. She is currently working on a numerical simulation of optical radiation force based on structured light.

Keisaku Yamane received his $\mathrm{PhD}$ from Kyoto University in 2005. He is an associate professor at Hokkaido University. His current research interests include ultrafast structured light and ultrafast physics. He is a member of OSA and JSAP.

Katsuhiko Miyamoto received his PhD from Tohoku Gakuin University in 2004. He is an associate professor at Chiba University. His research interests include terahertz photonics and nonlinear optics. He is a member of OSA, JSAP, and LSJ.

Takashige Omatsu received his $\mathrm{PhD}$ from the University of Tokyo in 1992. He was appointed as a professor at Chiba University in 2007. He is currently working on structured light and optical materials processing. He is also serving as an editor-in-chief, OSA continuum, and a steering committee of CLEO-Pacific Rim. He was elected a fellow of OSA, JSAP, and SPIE. 